\title{
Cooperative Transmit-Power Estimation in MANETs
}

\author{
Ivan Wang-Hei Ho* ${ }^{*}$ Bong Jun Ko ${ }^{\dagger}$, Murtaza Zafer ${ }^{\dagger}$, Chatschik Bisdikian ${ }^{\dagger}$, and Kin K. Leung* \\ "Department of Electrical and Electronic Engineering, Imperial College London, UK \\ †IBM T. J. Watson Research Center, USA \\ *\{wh.ho, kin.leung\}@imperial.ac.uk, ‘\{bongjun_ko, mzafer, bisdik\}@us.ibm.com
}

\begin{abstract}
Transmit-power estimation is an important part in power-aware designs of Mobile Ad-hoc Networks (MANETs). In this paper, we consider the cooperation among multiple monitor-nodes to estimate the transmit power of other nodes. Utilizing a geometric approach, we characterize the theoretical performance of such cooperative monitoring schemes and propose transmit-power estimation techniques with different number of cooperating nodes. We introduce the novel concept of confidence region that provides a fundamental confidence level for the accuracy of the power estimation and enables the development of techniques for allocating network monitors. Finally, we present a simple, distributed cooperative estimation scheme for a large-scale wireless network and give illustrative simulation results to quantify its performance.
\end{abstract}

Keywords - Mobile Ad-hoc Network, Network Monitoring, Cooperative Transmit-Power Estimation.

\section{INTRODUCTION}

We consider a wireless network and address the problem of estimating the transmission power of a node based on the received power levels at a set of monitors. Transmit-power estimation has many applications in distributed wireless networking environments such as MANETs. For instance, transmit-power estimation can be utilized for monitoring abnormal or malicious behavior of nodes that transmit at excessive power levels, thereby, causing signal jamming-attack [1] or channel capturing [2]. Transmit-power estimation may also be used to support power-aware operations in MANETs, such as transmission power control, cognitive radio, and power-aware routing [3] in heterogeneous environments where certain nodes may not be appropriately set to notify others of their own transmission power.

Despite its importance and wide applicability, there has been only a limited number of studies on the problem of transmit-power estimation. There are a number of works on mobile node positioning based on Received Signal Strength (RSS) measurements [4 - 6], but all of them assume the transmit power is known. In [7], the authors consider a cognitive radio setup and study the estimation of a node's position and transmit power using an ad-hoc optimization approach, while our work here explores the problem from a geometric perspective and characterizes the performance gain of the collaboration with a small number of monitoring nodes. Recently, the area of network monitoring of nodes' misbehavior in MAC layer has attracted considerable research efforts $[8,9]$. Regarding the physical (PHY) layer, there has been a body of work dealing with the management of radio resources (e.g., transmit power [2], rate [10], channels [11], etc.) for optimal network operation. However, unlike the MAC layer, there are limited studies covering diagnosis problems of misbehavior at the physical layer, such as mis-configured transmission power of mobile nodes.

In this paper, we explore the issue of transmit-power estimation and characterize the theoretical performance of schemes that utilize a novel collaboration of network monitoring nodes. More specifically, we address the following question: Suppose that a number of monitor-nodes measure the received power levels from a node at an unknown location and distance. If that node's transmit power has to be estimated solely based on the signal strength observed by the network monitors, to what degree can the cooperation of multiple, mutually-trusting nodes improve the accuracy of the estimation?

In answering the above question, we provide a theoretical analysis on bounds and their accuracy of estimating transmit power, as well as the gain achievable through the cooperation of multiple monitoring nodes. In particular, we consider a conservative estimation based on a geometric approach, which means that the estimation is made by finding the lower bound of the transmission power imposed by geometric constraints of nodes without any knowledge on the statistical characteristics of the transmission power. Our analytical results reveal that a simple, "light-weight" model of monitor cooperation can be a powerful tool that can improve the quality of the estimation by an order of magnitude. The results derived here based on a deterministic signal propagation model serve as benchmark estimates for studying cooperation of monitors under more advanced signal propagation models, and in facilitating the design of practical, distributed, monitoring mechanisms.

Our main contributions in this paper are as follows:

- We propose a simple, distributed cooperation model for multiple monitors and show that even with a small number of cooperating nodes, the quality of estimation can be significantly improved.

- We provide analytical results on the accuracy and the performance gain of transmit-power estimation when two and three monitors cooperate.

- We introduce the concept of confidence region which quantifies the accuracy of an estimation scheme. It can be utilized in unique ways in allocating network monitors for coverage.

Regarding the problem at hand, it may appear, at first glance, that the transmit-power estimation problem is similar to the node localization problem $[4-6,12]$, since the latter also typically utilizes geometric analysis based on distance metrics from multiple, cooperating, probing stations (or monitors). However, as mentioned earlier, the fundamental difference and the uniqueness of the transmit-power estimation problem comes from the fact that both the transmit power as well as the distance information between the monitored node and monitoring nodes are unknown. The ramification of this difference is highlighted in our analysis results on the estimation accuracy of three monitors, as discussed in Section 4. In particular, from localization techniques $[4-6,12]$, three cooperating probes can give the exact location of the monitored node under the deterministic signal propagation model as long as the three probes are not co-linearly located, whereas, it turns out that in our context, 
cooperation of three monitors does not guarantee a full accuracy of the transmit-power estimation. Interestingly enough, we show that full accuracy is achieved when three monitors are co-linearly located!

The rest of the paper is organized as follows. Section 2 provides the system model and performance metrics. Section 3 and 4 present the analysis results for the cases of two and three monitors' cooperation. Section 5 discusses approaches to cooperative monitoring in large-scale networks and related issues, and also presents performance evaluation through simulations. Finally, Section 6 concludes the paper.

\section{SYSTEM MODEL AND PERFORMANCE METRICS}

\section{A. System Model}

We consider a wireless ad-hoc network consisting of a set of nodes, $N$, which is a union of two subsets, $M$, the set of monitoring nodes (or "monitors"), and $T$, the set of monitored nodes (or "transmitters"). For the sake of simplicity, we assume the sets $M$ and $T$ are disjoint; in practice this need not be the case and the results presented in this paper still apply.

Let $P_{i}$ denote the (unknown) transmit power of node $i$ in $T$, and $P_{i m}$ the received power at a monitoring node $m$ in $M$. Also, we denote by $d_{i j}$ the distance between two nodes $i$ and $j$ in $N$; for notational brevity, unless necessary, we will drop the explicit reference to node $i$, e.g., we will write $d_{m}$ (omitting the index for node $i$ ) for the (unknown) distance between node $i$ and monitoring node $m$. We do not make any assumption about the statistical characteristics (e.g., mean, variance, distribution, etc.) of the transmission power.

We say that a monitoring node $m$ is able to monitor a node $i$ in $T$ if $P_{i m}>R x_{t h}$, where $R x_{t h}$ is a constant for the minimum received power threshold, which enables a monitoring node to identify the corresponding transmitter of the received signal ${ }^{1}$. We denote by $M_{i} \subset M$ the set of monitoring nodes in $M$ that can monitor node $i$.

Our problem is to estimate the unknown transmit power $P_{i}$ of a node $i$ in $T$, given the set of received power levels $\left\{P_{i m}: m \in M_{i}\right\}$. Our goal is to investigate a novel use of cooperation between monitors in estimating the transmit power of node $i$. To proceed, we first introduce the cooperation model of monitoring nodes.

We say that a pair of monitors, $m$ and $n$ in $M$, can cooperate with each other to estimate the transmit power of a node $i$ in $T$ under the following three conditions:

(C1) Both $m$ and $n$ can monitor $i$ (i.e., $m \in M_{i}$ and $n \in M_{i}$ );

(C2) The distance $d_{m n}$ between $m$ and $n$ is known; and

(C3) Nodes $m$ and $n$ can exchange their respective received power information, $P_{i m}$ and $P_{i n}$, with each other.

Note that in the above model we do not require the geometric coordinates of the monitors to be known; instead, we only need to know the distance between the monitors ${ }^{2}$. Note also that (C3) is an optional condition that enables the fully distributed implementation of our cooperative estimation scheme and is not relevant to the foregoing analysis.

\footnotetext{
1 Identifying the transmitter can be done in practice by reading some information encoded in the signal (e.g., identifiers in MAC, IP, or higher layer). We do not assume, however, any particular method of transmitter identification in this paper.

${ }_{2}^{2}$ We do not assume a particular method for obtaining the distance information between a pair of monitors. One possible way to obtain it would be to equip each monitor with a GPS device to find out their positions in outdoor environments. Another way could be to let the two monitors exchange their respective transmit power information and derive their inter-distance according to the signal propagation model.
}

We assume a deterministic signal propagation model, $P_{i m}=k \cdot P_{i} / d_{i m}{ }^{\alpha}$ [13], where $d_{i m}>1$ is the (unknown) distance between nodes $i$ and $m, k$ and $\alpha$ (path-loss exponent) are known constants. We make use of this deterministic propagation model to obtain a fundamental understanding of the achievable performance gain through cooperation of monitors, and to establish a benchmark for future studies that consider more advanced signal propagation models, such as incorporating stochastic propagation behavior due to log-normal shadowing [13] in multi-path fading channel and unknown path-loss exponent.

\section{B. Performance Metrics}

We consider a conservative transmit-power estimation scheme: Given the observations $\left\{P_{i m}: m \in M_{i}\right\}$ and $\left\{d_{m n}\right\}$ for cooperating monitor pairs $(n, m) \in M_{i} \times M_{i}$, we derive a (tight) lower bound for the possible value of the transmit power $P_{i}$ of monitored node $i$, and use this lower bound as our estimated transmit power $P_{i}{ }^{*}$ - in other words, we do not allow over-estimating the actual transmit power.

We analyze the performance of the cooperative estimation via the following parameters:

Estimation accuracy ( $r)$ : is defined as the ratio of estimated to actual transmit power of the monitored node $i$.

$$
r_{i}=\frac{P_{i}^{*}}{P_{i}}
$$

The accuracy $r$ captures the quality of an estimation scheme. For an effective power estimation scheme, it would be desirable to have $r$ as close to 1 as possible.

Cooperative gain $(\mathbf{g})$ : is defined as the ratio of estimated power by multiple cooperating monitors to that with noncooperating monitors.

$$
g_{i}=\frac{P_{i}^{*}}{\max _{m \in M_{i}}\left\{P_{i m}\right\}}
$$

where the denominator gives the best possible estimation by a set of non-cooperating monitors, when the only available information to the monitors is the set of received powers. This metric captures the performance gain achieved by the cooperation of monitors compared to the non-cooperative monitoring case.

Confidence region $(\boldsymbol{R}(\boldsymbol{r}))$ : is defined as the geometrical region of the location of a monitored node, such that within this region the power estimation accuracy is at least at value $r$. The confidence region gives the area that a set of monitors "covers" for a specified minimum estimation accuracy. One would like to have it as large as possible in order to maximize the coverage of the given monitoring resources.

In the subsequent sections, we provide the details of the power-estimation scheme and its analysis. We first study the two-monitor case followed by the three-monitor case. Finally, we present a simple, cooperative estimation scheme for a large-scale network.

\section{TWO-MONITOR COOPERATION}

Consider the scenario in Figure 1 where two monitors, nodes 1 and 2, cooperate to estimate the transmit power $P_{i}$ of node $i$. Let $d_{1}$ and $d_{2}$ denote the (unknown) distance between node $i$ and monitors 1 and 2 , respectively. Also, let $d_{12}$ denote the known distance between nodes 1 and 2 .

We use the triangular inequality, $d_{1}+d_{2} \geq d_{12}$, to obtain a lower bound on $P_{i}$, which we will use as the estimate $P_{i}^{*}$ of $P_{i}$ for the two-monitor cooperation case. Using the signal propagation model this gives, 


$$
d_{1}+d_{2}=\left(\frac{k P_{i}}{P_{i 1}}\right)^{\frac{1}{\alpha}}+\left(\frac{k P_{i}}{P_{i 2}}\right)^{\frac{1}{\alpha}} \geq d_{12} \Rightarrow P_{i} \geq P_{i}^{*},
$$

where $P_{i}^{*}$, our transmit-power estimate, equals

$$
P_{i}^{*}=\left(\frac{d_{12}}{\left(k / P_{i 1}\right)^{\frac{1}{\alpha}}+\left(k / P_{i 2}\right)^{\frac{1}{\alpha}}}\right)^{\alpha}
$$

From the definition of $P_{i}^{*}$, it follows that $r=P_{i}^{*} / P_{i} \leq 1$. The bound (and power estimate) $P_{i}^{*}$ of $P_{i}$ is a tight bound in the sense that there exists a location of the monitored node $i$ (relative to the locations of monitoring nodes) which results in the bound $P_{i}{ }^{*}$ to equal the actual transmit power $P_{i}$. This can be verified by looking at the trajectory of the possible location of node $i$, as shown next.

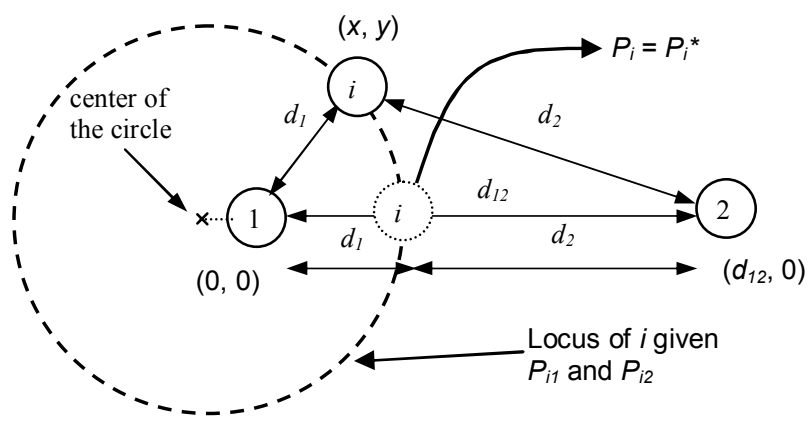

Figure 1. Two-monitor case.

Given two distinct received signal power levels observed by a pair of monitors, the possible locations of the monitored node $i$ has a circular trajectory (locus).

Proposition 1. Given the received power levels $P_{i 1}$ and $P_{i 2}$, where $P_{i 1} \neq P_{i 2}$, of a monitored node $i$ at a pair of monitors 1 and 2 , the possible locations of $i$ lie on a circle.

Proof. Given $P_{i 1}$ and $P_{i 2}$, using the signal propagation model, the ratio $c_{1}$ of $d_{1}$ to $d_{2}$ is given by

$$
\frac{d_{1}}{d_{2}}=\left(\frac{P_{i 2}}{P_{i 1}}\right)^{\frac{1}{\alpha}}=c_{1}
$$

With regard to the coordinate system in Figure 1, we have

$$
\frac{d_{1}^{2}}{d_{2}^{2}}=\frac{x^{2}+y^{2}}{\left(x-d_{12}\right)^{2}+y^{2}}=c_{1}^{2}
$$

which is the equation of the circle $\mathrm{C} 1$ :

$$
\mathrm{C} 1:\left(1-c_{1}^{2}\right) x^{2}+\left(1-c_{1}^{2}\right) y^{2}+2 c_{1}^{2} d_{12} x-c_{1}^{2} d_{12}^{2}=0
$$

centered at $\left(\frac{-c_{1}^{2} d_{12}}{1-c_{1}^{2}}, 0\right)$ and having radius $\frac{c_{1} d_{12}}{1-c_{1}^{2}}$.

Note that the center of the circle lies on the same line as monitors 1 and 2 (but not at the positions of monitor 1 or monitor 2). Instead, its center is located outside the triangle formed by nodes 1, 2 and $i$ as shown in Figure 1.

Now, among all the possible locations of node $i$ on circle $\mathrm{C} 1$, if $i$ was actually on the straight line joining monitors 1 and 2 (see the dotted node $i$ location in Figure 1), the corresponding transmit power $P_{i}$ which gives the received powers $P_{i 1}$ and $P_{i 2}$ is equal to the lower bound computed in (1); i.e., for this location $P_{i}=P_{i}^{*}$, and our estimation scheme achieves an accuracy of 1 . For the special case when $P_{i 1}=P_{i 2}$, the trajectory of $i$ becomes the perpendicular bisector of the straight line joining monitors 1 and 2 .

The accuracy and gain (defined in Section 2.B) of twomonitor cooperation are given as follows:

$$
\begin{gathered}
r=\left(\frac{d_{12}}{d_{1}+d_{2}}\right)^{\alpha} \\
g=\frac{P_{i}^{*}}{P_{i 1}}=\frac{1}{k} \cdot\left(\frac{d_{1} d_{12}}{d_{1}+d_{2}}\right)^{\alpha}
\end{gathered}
$$

where in (3) we assume, without loss of generality, that $P_{i 1} \geq$ $P_{i 2}$. Let us first investigate $g$ to see how substantial gains can be achieved; consider the case $P_{i 1}=P_{i 2}$ which reduces (3) to the

form $(1 / k) *\left(d_{12} / 2\right)^{\alpha}$. Assuming $d_{12}$ greater than 2 , an exponential growth in $g$ with respect to the distance between the monitoring nodes can be obtained. In fact, such substantial cooperation gain is also observed in simulations as discussed later in Section 5. Note that it can be easily seen that $P_{i}{ }^{*} \geq \max \left(P_{i 1}, P_{i 2}\right)$, and hence, the gain for two-monitor cooperation is always greater than or equal to 1 .

An essential feature of the above results is that the accuracy increases as the distance between monitors $\left(d_{12}\right)$ increases. This implies that the cooperation of monitors can achieve better estimation results when the diversity of the individual monitors' observations (due to the geometric separation of monitors) can be exploited.

A consequence of (2) for the estimation accuracy is an elliptical confidence region of two-monitor cooperation: Specifically, (2) implies that as $d_{1}$ and $d_{2}$ vary but the sum $d_{1}$ $+d_{2}$ remains constant so does the value of the accuracy $r$ too. Therefore when $r$ is fixed, and hence the sum $d_{1}+d_{2}$ is constant, it follows that the node $i$ lies along an ellipse with the two monitors 1 and 2 being its foci (see Figure 2). From properties of ellipses, the sum that $d_{1}+d_{2}=2 \gamma$ represents the major axis of the ellipse. Therefore, $r=\left(d_{12} /\left(d_{1}+d_{2}\right)\right)^{\alpha}=(2 c / 2 \gamma)^{\alpha}=(c / \gamma)^{\alpha}$, which is a constant.

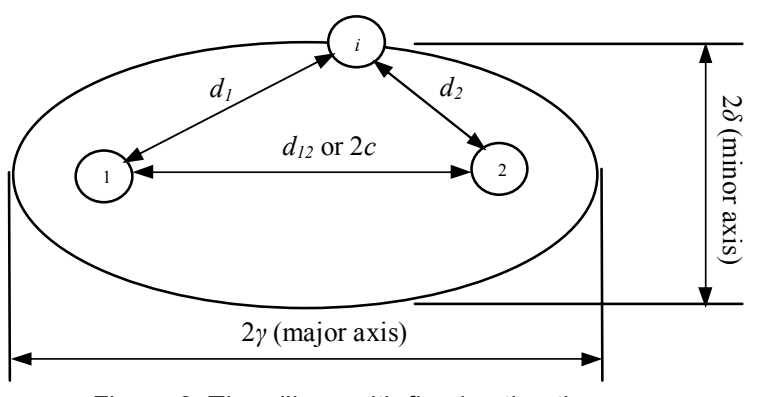

Figure 2. The ellipse with fixed estimation accuracy.

Since at any point inside the ellipse $d_{1}+d_{2} \leq 2 \gamma$, it follows that the area enclosed by the ellipse defines the confidence region such that the accuracy of the estimated transmit power is greater than or equal to $(c / \gamma)^{\alpha}$. Furthermore, given the elliptical confidence region, if we assume that the location of node $i$ is uniformly distributed within it, the expected accuracy $E[r]$ is given by, e.g., for $\alpha=2$ :

$$
\begin{aligned}
E[r] & =\frac{1}{A_{c}} \int_{c}^{\gamma} r d A=\frac{1}{\pi \gamma \sqrt{\gamma^{2}-c^{2}}} \int_{c}^{\gamma}\left(\frac{c}{x}\right)^{2} d A \\
& =-\frac{c^{2}}{\gamma^{2}}+\frac{2 c^{2}}{\gamma \sqrt{\gamma^{2}-c^{2}}} \ln \left(\frac{\gamma+\sqrt{\gamma^{2}-c^{2}}}{c}\right),
\end{aligned}
$$

where $A_{c}=\pi \gamma \sqrt{\gamma^{2}-c^{2}}$ is the area of the ellipse.

It is interesting to observe that both the minimum accuracy and the expected accuracy $E[r]$ of the elliptical confidence region can be expressed as a function of $(c / \gamma)$, i.e., the eccentricity of the ellipse. In geometrical terms, the eccentricity of an ellipse is a value between 0 and 1 that determines the shape of the ellipse; when this value is close to 0 the ellipse becomes circular, while when close to 1 it becomes "flat". This translates to the fact that the 
eccentricity (thus the shape of the elliptical confidence region) is what invariantly determines the minimum and expected accuracy of the confidence region, regardless of the size of the region. Figure 3 plots $E[r]$ as the function of $(c / \gamma)$ for $\alpha=2,3$, and 4 , which shows the expected accuracy increases as the eccentricity increases.

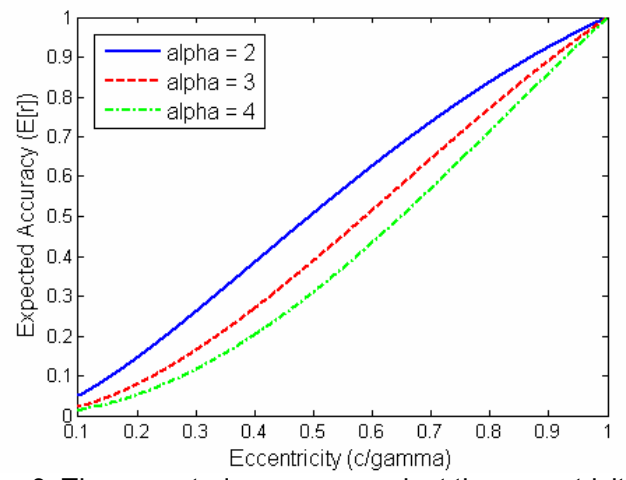

Figure 3 . The expected accuracy against the eccentricity of the elliptical confidence region with $\alpha$ equals 2,3 , and 4 .

The elliptical confidence region can be used in a novel way to approximate the number of monitors required to achieve a certain degree of accuracy for a given monitored area. Specifically, suppose we utilize pairs of monitors with fixed $d_{12}$ to cover a certain area for transmit-power monitoring. Given an (minimum or expected) accuracy requirement $r$, one can calculate the area of the corresponding confidence region given by a pair of monitors with inter-distance $d_{12}$ (given by $A_{c}=\pi \gamma \sqrt{\gamma^{2}-c^{2}}$ ). Using this, the minimum number of monitor pairs with twomonitor cooperation that would be needed to cover a geographic region of area $A_{d}$ is approximately $A_{d} / A_{c}$.

\section{THREE-MONITOR COOPERATION}

We now investigate the three-monitor cooperation case, focusing on three monitors, 1, 2, and 3, and the monitored node $i$ (see Figure 4). Again, we assume the transmit power of node $i$ and its distances to monitor-nodes are all unknown.

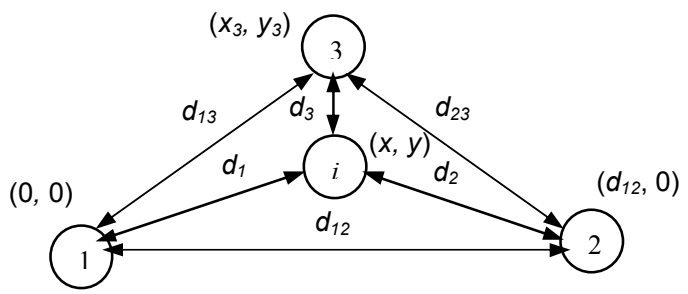

Figure 4. Three-monitor case.

The cooperation of three monitors can be divided into two cases: first, when all three monitors are in full cooperation, i.e., the inter-distances between all pairs of monitors are known, and second, when the distances between only two pairs of monitors are known (the case when the distance between only one pair of monitors is known degenerates to a two-monitor cooperation model).

\section{Case 1: Full Cooperation}

Suppose that the distances $d_{12}, d_{23}$, and $d_{13}$ are all known. Since all three distances are known, the relative coordinates of the monitors with respect to each other are also known. Although the distances between the monitored and monitoring nodes are unknown, the ratios of $d_{1}, d_{2}$, and $d_{3}$ can be obtained as follows;

$$
\frac{d_{1}}{d_{2}}=\left(\frac{P_{i 2}}{P_{i 1}}\right)^{\frac{1}{\alpha}}=c_{1}, \frac{d_{1}}{d_{3}}=\left(\frac{P_{i 3}}{P_{i 1}}\right)^{\frac{1}{\alpha}}=c_{2}, \frac{d_{2}}{d_{3}}=\left(\frac{P_{i 3}}{P_{i 2}}\right)^{\frac{1}{\alpha}}=c_{3}=\frac{c_{2}}{c_{1}}
$$

Given these three ratios, it follows from Proposition 1 that we can obtain three equations of circles which represent the loci of the monitored node. It seemingly appears that the three variables $x$ ( $x$-coordinate of $i), y$ ( $y$-coordinate of $i$ ) and $P_{i}$ can be solved for with these three equations. However, due to the triangular dependency of $c_{3}$ on $c_{2}$ and $c_{1}$, it can be shown that the three circles intersect at two points as shown in Figure 5, which implies that there are two possible locations for the position of monitored node and thus the corresponding transmit powers level.

Theorem 1. Suppose $d_{12}, d_{23}$, and $d_{13}$ are known. If $c_{1}, c_{2}$, and $c_{3}$ are not all equal to 1 , there are two possible solutions for the position of the monitored node $i$.

Due to space limitations, the detailed proof of Theorem 1 is not included here (see [14] for the proof), but an intuitive illustration of the proof is as follows: Two circular loci of $i$ (Circles 1 and 2) obtained from any two pairs of monitors have two intersection points, and the third circular locus (Circle 3 ) from the third monitor pair can be proved to belong the family of circles that pass through the intersections of Circles 1 and 2 due to the triangular dependency of $c_{3}$ on $c_{2}$ and $c_{1}$.

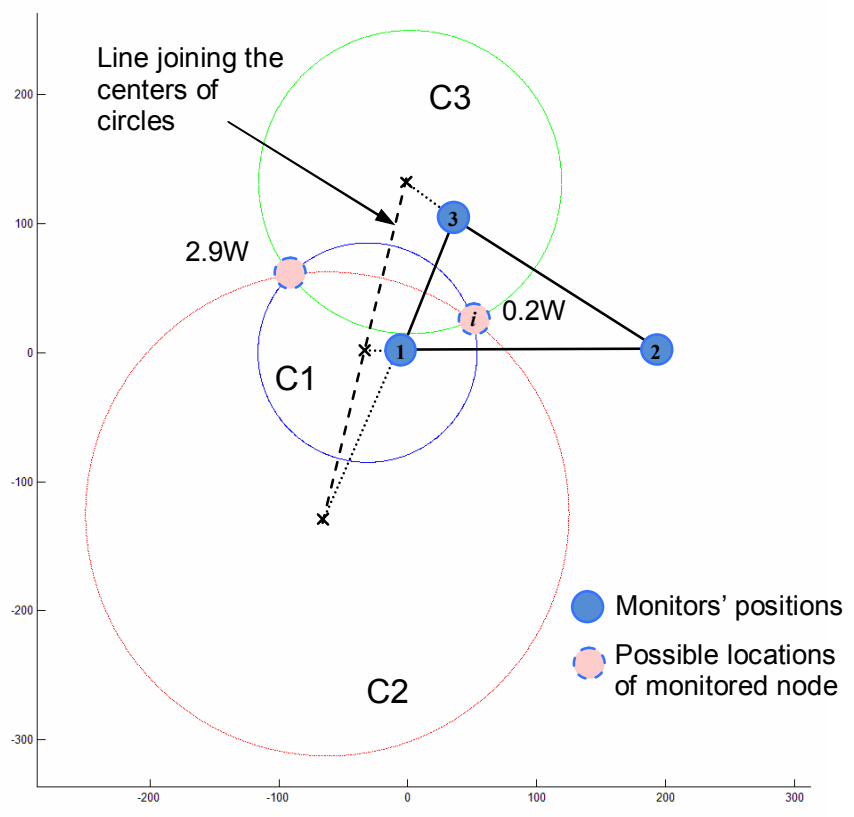

Figure 5. Graphical illustration of the intersections of the three circles that indicate the possible locations of the monitored node.

Given Theorem 1, let the coordinates of the centers of Circle $1(\mathrm{C} 1)$ and Circle $2(\mathrm{C} 2)$ be $\left(x_{C 1}, y_{C 1}\right)$ and $\left(x_{C 2}, y_{C 2}\right)$ respectively. Let $R_{1}$ and $R_{2}$ denote the radius of $\mathrm{C} 1$ and $\mathrm{C} 2$ respectively, and $d$ be the distance between the centers of $\mathrm{C} 1$ and $\mathrm{C} 2$. Then, the solutions for the locations of the monitored node $i$ are

$$
\begin{aligned}
& x_{i}=\frac{x_{C 1}+x_{C 2}}{2}+\frac{\left(x_{C 2}-x_{C 1}\right)\left(R_{1}^{2}-R_{2}^{2}\right)}{2 d^{2}} \pm \frac{y_{C 2}-y_{C 1}}{2 d^{2}} \sqrt{\left(\left(R_{1}+R_{2}\right)^{2}-d^{2}\right)\left(d^{2}-\left(R_{2}-R_{1}\right)^{2}\right)} \\
& y_{i}=\frac{y_{C 1}+y_{C 2}}{2}+\frac{\left(y_{C 2}-y_{C 1}\right)\left(R_{1}^{2}-R_{2}^{2}\right)}{2 d^{2}} \mp \frac{x_{C 2}-x_{C 1}}{2 d^{2}} \sqrt{\left(\left(R_{1}+R_{2}\right)^{2}-d^{2}\right)\left(d^{2}-\left(R_{2}-R_{1}\right)^{2}\right)}
\end{aligned}
$$

Thus, $P_{i}=\frac{P_{i 1} \cdot d_{1}^{\alpha}}{k}=\frac{P_{i 1}\left(x_{i}^{2}+y_{i}^{2}\right)^{\alpha / 2}}{k}$

Between the above two solutions, we select the smaller value of $P_{i}$ as the estimate $P_{i}{ }^{*}$. Figure 5 shows two possible locations of the monitored node and their corresponding transmit powers, $2.9 \mathrm{~W}$ or $0.2 \mathrm{~W}$. In this case, the transmitpower estimation scheme picks $P_{i}{ }^{*}$ as $0.2 \mathrm{~W}$.

We notice that, although there are two possible positions of the monitored node based on the observations from three 
monitors, we can still get a unique solution for $P_{i}$ if in fact the three monitors are co-linearly located.

Corollary 1. With three monitors located along a straight line, there is a unique solution for the transmit power of the monitored node, and this solution equals the actual transmit power of the monitored node.

The sketch of the proof is as follows (see [14] for the detailed proof). If the three monitors are placed along a line, all the centers of the three circles drawn by the ratios $c_{1}, c_{2}$, and $c_{3}$ lie on this line as well, and the two intersections of these circles are located in symmetric positions relative to the line. Therefore the two possible locations of the monitored node have the same distance from each monitor, and hence result in a unique value of $P_{i}{ }^{*}$ which is equal to $P_{i}$.

The above optimal placement of three-monitors facilitates the design of a monitoring network with full accuracy under the deterministic power propagation assumption. More specifically, we can place monitoring nodes in a two dimensional grid topology, in which a monitoring node cooperates with at least two other monitors along the same (horizontal or vertical) line. The important decision then in designing such a distributed monitoring infrastructure is to consider the coverage area and the size of the grid such that any monitored node in the network can be monitored by at least three monitors in cooperation.

\section{Case 2: Partial Cooperation}

Without loss of generality, assume that $d_{12}$ and $d_{13}$ are known but $d_{23}$ is unknown. If we fix the relative coordinates of nodes 1 and 2 (according to $d_{12}$ ), we are uncertain about the position of monitor 3 . Therefore, in general, the partial cooperation of three monitors with the distance between a pair of monitors missing can only be handled in an equivalent manner to the case when there are two pairs of monitor nodes $(1,2)$ and $(1,3)$, with each pair cooperating independently in a two-monitor cooperation model.

However, we can make a better estimation when the following assumption holds: the distance between two monitors $d_{n m}$ is known if $d_{n m}<d_{\max }$, and is unknown if $d_{n m}$ $\geq d_{\max }$. This condition reflects the geographic constraints in which two monitors can find out their inter-distance only if they are less than $d_{\max }$ apart, e.g., by measuring the transmit power of their partner monitor. In this case, in reference to Figure 4, node 3 can be located at any point on the circle centered at monitor 1 with radius $d_{13}$, where $d_{23} \geq d_{\max }$. Again, different possible locations of monitor 3 correspond to different solutions for the position and transmit power of the monitored node. However, since, under our conservative estimation model, we always select the smallest possible solution of $P_{i}$ as our estimate $P_{i}{ }^{*}, P_{i}$ reaches its lower bound when monitor 3 lies on the boundary of the transmission range of monitor 2, where $d_{23}=d_{\text {max }}$. Using (4), we can then find the estimate $P_{i} *$ by setting the unknown $d_{23}$ to $d_{\max }$.

Having estimated $P_{i}{ }^{*}$, it is worthwhile comparing the confidence regions for 1-monitor, 2-monitor and 3-monitor cases. It turns out that deriving a closed formed expression for the confidence region in the 3 -monitor case is mathematically intractable, but nevertheless, we can still derive plots numerically. Figure 6 gives an illustrative plot where we have three monitors placed at the angular points of an equilateral triangle, and we draw the confidence region for estimation accuracy $r=0.5$. The two small circles (appearing as dots) at the bottom two monitors' positions denote the region when only each individual monitor performs estimation without cooperation, the ellipse in the lower part of the figure gives the region when 2-monitor cooperation is utilized, and the larger circle is with full 3monitor cooperation. It can be seen from the figure that the confidence region of a single monitor is covered by that of two monitors, which in turn being covered by that of three monitors. It also validates our expectation that the cooperative gain increases with the number of cooperating monitors.

We conclude this section by briefly discussing the case when more than three monitors cooperate for estimating the transmit power of a node. In this case, if the combinations of inter-monitor distances allow the formation of two independent triangles of fixed relative coordinates of monitoring nodes (given that the monitoring nodes are not placed on an arc of a circle), we can make use of the ratios among $d_{1}, d_{2}, d_{3}$ and $d_{4}$ (distances between the monitored node and the four monitors) to determine the exact location of the monitored node, and hence its transmit power as well. This is because the two possible locations given by one triangle of monitoring nodes do not have the triangular dependency on the other triangle. Therefore, the corresponding circles intersect at one point instead of two points, providing the actual location and the transmit power of the monitored node under the deterministic signal model.

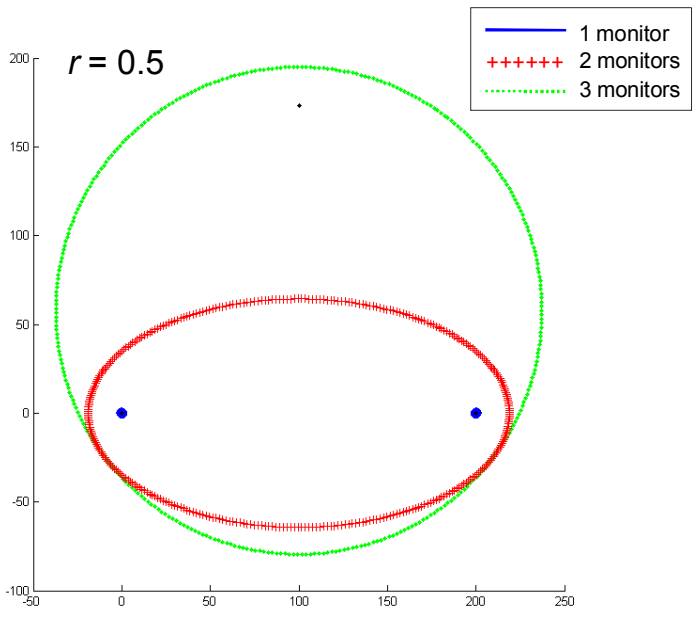

Figure 6. Confidence regions for a fixed accuracy with 1,2 , and 3 cooperative monitors.

\section{MAXIMUM PAIRWISE COOPERATION POLICY (MAXPAIR) FOR LARGE-SCALE NETWORK}

In a large-scale ad-hoc network, implementing threeand four-monitor cooperation for optimal estimation quality can be difficult due to required monitor density and node positioning as well as the computational complexity. In such scenarios, it would be more suitable to have a simple yet powerful cooperation estimation scheme. Towards this goal, we utilize the two-monitor case discussed in Section 3 to obtain a simple and practical cooperative estimation policy for large-scale networks. We refer to it as the Maximum Pairwise Cooperation Policy (MaxPair) and evaluate its performance through simulations.

In MaxPair, monitors cooperate in a pairwise manner when they are within the transmission range of each other. Considering a monitored node, multiple cooperating monitor pairs would produce multiple estimations of the transmit power. In such a case, MaxPair selects the maximum of these estimates as the final estimated transmit power of the monitored node. In other words, let $d_{\max }$ be the transmission range and $M$ be the set of monitors, then for MaxPair, $P_{i} *=$ $\max \left(P_{i}^{*}(a, b)\right) \forall a, b \in M_{i}$, where $|a-b| \leq d_{\text {max }}$ and $a \neq b$.

Since the max operator results in larger values the larger 
the set, over which it operates, the MaxPair power estimate $P_{i}$ * increases each time a new monitor is added (at least when the location of the "old" monitors does not change). In other words, if $P_{i, n} *$ is the estimation accuracy of MaxPair when there are $n$ nodes, then $P_{i, n}{ }^{*} \leq P_{i,(n+1)} *$, which in turn, implies that: $r_{n} \leq r_{n+1} \leq 1$. The RHS of the latter expression follows from the fact that $P_{i}^{*}(a, b) \leq P_{i}$ for any pair $a$ and $\mathrm{b}$ of monitors.

We now present simulation results to show the performance of MaxPair policy. First, we compare the performance of MaxPair with three-monitor cooperation. As a microscopic scenario, we place three monitors at the vertices of an equilateral triangle and randomly place transmitter nodes within the area defined by a circle from the centroid of the triangle. For transmit-power estimation we follow the two schemes: 1) full three-monitor cooperation as discussed in Section 4; and 2) MaxPair policy (with three pairs).

Figure 7 plots the estimation accuracy versus the monitored area (area of the circle within which the transmitter is randomly placed). As we expect from our results of confidence region in Section 3, the estimation accuracy decreases as the monitored area grows larger; interestingly however, the three-monitor case out-performs MaxPair only by an accuracy of around 0.1 .

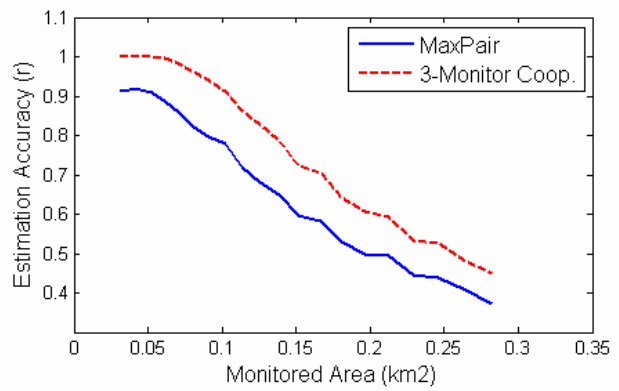

Figure 7. Estimation accuracy of MaxPair and the three-monitor cooperation model against the monitored area.

We next consider a large-scale network simulation where we randomly place 1000 transmitter nodes in a $1 \times 1$ $\mathrm{km}^{2}$ domain. Monitoring nodes are also randomly placed with the monitor density $(\lambda)$, measured as the number of monitors per unit area, is increased from 1/100000 (with 10 monitors) to $1 / 10000$ (with 100 monitors). We set the transmission range to $250 \mathrm{~m}$ which implies that monitors within the transmission range of each other cooperate in pairwise manner for MaxPair.

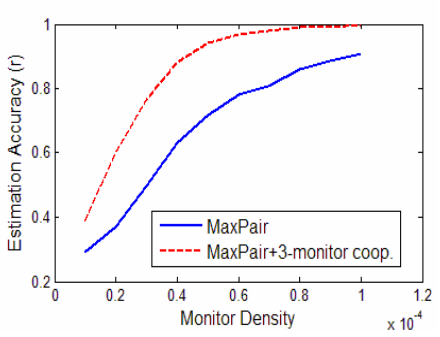

(a)

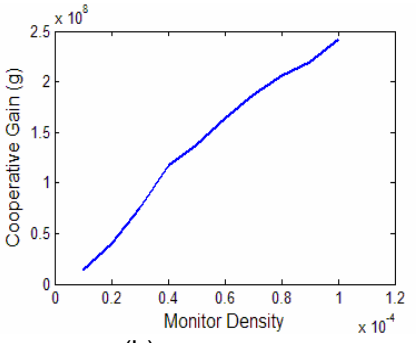

(b)
Figure 8. a) Estimation accuracy of MaxPair and the hybrid model; and b) cooperative gain of MaxPair against monitor density.

Figures $8(\mathrm{a})$ and 8 (b) plot the average accuracy and the gain of the MaxPair policy. As is evident from the plots, both the estimation accuracy $r$ and cooperative gain $g$ of MaxPair increase as the monitor density increases. Also, the gain $g$ is by a few orders of magnitude, thereby showing that the cooperation is quite effective for transmit-power estimation. In addition to MaxPair, we also consider a hybrid cooperation model, MaxPair plus three-monitor cooperation, to see how much the accuracy improves with more cooperating nodes. In the hybrid case, if three monitors are within the transmission range of each other, they will cooperate according to the three-monitor cooperation model (Case 1) described in Section 4. We can see from Figure 8(a) that the difference in accuracy between the two cases is small, especially at the two ends. This is because, when the monitor density is low, it is difficult to have randomly placed three monitors inter-connected to cooperate. While at higher monitor density (e.g., $\lambda=1 / 10000)$, MaxPair solely can already achieve a satisfactory level of estimation accuracy $(r$ $>0.9$ ), and the accuracy gain of the hybrid case at this density level is less than $10 \%$.

\section{CONCLUSION AND FUTURE WORK}

In this paper, we have established the positive impact that the collaboration of a small number of monitors brings to transmit-power estimation. We analytically studied the achievable performance of the cooperative estimation at a fundamental level by considering a simple cooperative model of multiple monitors. Our analytical results, demonstrated in terms of characteristics such as estimation accuracy, gain, and confidence region, suggest that the cooperation of small number of network monitors can be a powerful tool for estimating unknown transmit-powers of other nodes in MANETs. We also proposed a simple and practical form of cooperative power estimation policy (MaxPair) for large-scale ad-hoc network and presented illustrative simulation results quantifying its performance. In the future, we intent to study collaborative power estimation with more elaborate system models including stochastic propagation models.

\section{ACKNOWLEDGEMENTS}

Research was sponsored in part by the U.S. Army Research Laboratory and the U.K. Ministry of Defence and was accomplished under Agreement Number 911NF-06-3-0001. The views and conclusions contained in this document are those of the author(s) and should not be interpreted as representing the official policies, either expressed or implied, of the U.S. Army Research Laboratory, the U.S. Government, the U.K. Ministry of Defence or the U.K. Government. The U.S. and U.K. Governments are authorized to reproduce and distribute reprints for Government purposes notwithstanding any copyright notation hereon.

\section{REFERENCES}

[1] W. Xu, W. Trappe, Y. Zhang, T. Wood, "The feasibility of launching and detecting jamming attacks in wireless networks," MobiHoc, 2005.

[2] I. W.-H. Ho, S. C. Liew, "Impact of Power Control on Performance of IEEE 802.11 Wireless Networks," IEEE Trans. on Mobile Computing, vol. 6, no. 11, 2007.

[3] S. Singh, M. Woo, and C. S. Raghavendra, "Power-Aware Routing in Mobile Ad Hoc Networks," ACM/IEEE Mobicom, 1998.

[4] K. W. Cheung, H. C. So, W.-K. Ma, and Y. T. Chan, "Received Signal Strength Based Mobile Positioning via Contrained Wighted Least Square," IEEE ICASSP, 2003. [5] J. Ash, L. Potter, "Sensor Network Localization via Received Signal Strength Measurements with Directional Antennas," the 42nd Annual Allerton Conference on Communication, Control, and Computing, 2004.

[6] C. Liu, K. Wu, and T. He, "Sensor Localization with Ring Overlapping Based on Comparison of Received Signal Strength Indicator," the 1st IEEE International Conference on Mobile Ad-hoc and Sensor Systems, 2004.

[7] S. Kim, H. Jeon, H. Lee, J. S. Ma, "Robust Transmission Power and Position Estimation in Cognitive Radio," ICOIN, 2007.

[8] P. Kyasanur, N. Vaidya, "Selfish MAC Layer Misbehavior in Wireless Networks," IEEE Trans. on Mobile Computing, vol. 4, no. 5, 2005.

[9] S. Radosavac, J. Baras, I. Koutsopoulos, "A Framework for MAC Protocol Misbehavior Detection in Wireless Networks," the 4th ACM workshop on Wireless security, 2005.

[10] Z. Wu, S. Ganu, I. Seskar and D. Raychaudhuri, "Experimental Investigation of Physical Layer Rate Control and Frequency Selection in 802.11 Based Ad-hoc Networks," E-WIND ACM Sigcomm Workshop, 2005.

[11] P. C. Ng, D. J. Edwards, and S. C. Liew, "Doubling Capacities by a Linkdirectionality-based Dual Channel MAC Protocol for IEEE 802.11 Ad-hoc Networks," IEEE Globecom, 2006.

[12] N. Patwari, J. N. Ash, S. Kyperountas, A. O. Hero, R. L. Moses, N. S. Correal, "Locating the Nodes: Cooperative Localization in Wireless Sensor Networks," IEEE Signal Processing Magazine, vol. 22, no. 4, 2005.

[13] T. S. Rappaport, Wireless Communications: Principles and Practice. Englewood Cliffs, NJ: Prentice Hall, 1996.

[14] I. W.-H. Ho, B. Ko, M. Zafer, C. Bisdikian, and K. K. Leung, "Cooperative Transmit-Power Estimation in MANETs," IBM Research Report, RC24353, 2007. 